This is the accepted manuscript made available via CHORUS. The article has been published as:

\title{
Direct observation of phase-sensitive Hong-Ou-Mandel interference
}

Petr Marek, Petr Zapletal, Radim Filip, Yosuke Hashimoto, Takeshi Toyama, Jun-ichi

Yoshikawa, Kenzo Makino, and Akira Furusawa

Phys. Rev. A 96, 033830 - Published 18 September 2017

DOI: 10.1103/PhysRevA.96.033830 


\title{
Direct observation of phase sensitive Hong-Ou-Mandel interference
}

\author{
Petr Marek, Petr Zapletal, and Radim Filip \\ Department of Optics, Palacký University, 17. listopadu 1192/12, 77146 Olomouc, Czech Republic
Yosuke Hashimoto, Takeshi Toyama, Jun-ichi Yoshikawa, Kenzo Makino, and Akira Furusawa
Department of Applied Physics and Quantum-Phase Electronics Center, School of Engineering, The University of Tokyo, \\ 7-3-1 Hongo, Bunkyo-ku, Tokyo 113-8656, Japan
}

\begin{abstract}
Quality of individual photons and their ability to interfere is traditionally tested by measuring the Hong-Ou-Mandel photon bunching effect. However, this phase insensitive measurement only tests the particle aspect of the quantum interference, leaving the phase sensitive aspects relevant for continuous variables processing out. To overcome these limitations we formulate a witness capable of recognizing both the indistinguishability of the single photons and their quality with regards to their continuous variables utilization. We exploit the conditional nonclassical squeezing and we show that it can reveal both the particle and the wave aspects of the quantum interference in a single set of direct measurements. Finally, we experimentally test the witness by applying it to a pair of independent single photons retrieved on demand.
\end{abstract}

PACS numbers: 42.50.Xa, 42.50.Ar, 42.50.Dv, 42.50.Ex

Quantum interference of individual photons is one of the fundamental keystones of quantum technology. The ability to recognize whether photons from different sources can interfere is therefore an important one. The basic tests use photon correlation measurements to ascertain presence of individual photons and two-photon bunching to verify their interference. The experimental test of the bunching effect traditionally employs the Hong-Ou-Mandel interference [1], in which the two individual photons are mixed on a balanced beam splitter and the resulting two mode field is measured by a pair of photon counters. The bunching manifests as the lack of coincidence counts - if the coincidence probability is below one half, the interference effect is nonclassical, i. e. incompatible with description using a mixture of classical waves or classical particles. Recent experiments for a wide range of experimental platforms safely observed low values of coincidence counts and thus demonstrated this fundamental nonclassical aspect [2].

For discrete variable quantum technology (DV) these features are sufficient. However, from the point of view of continuous variables (CV) and the more general framework of hybrid quantum technology [3-6], these experiments quantify only the particle nature of the interference and ignore the vacuum-related phase sensitive aspects [7] of the photon bunched state. They also avoid the issues of coupling single photons with CV states, such as mode overlap with the local oscillator. The full picture of two-photon interference can be obtained by replacing the photon counters with homodyne detection and performing a full tomography $[8,9]$. The reconstructed density matrix can then be analyzed in order to visualize and evaluate the interference caused by the the indistinguishability. The drawback to this tomographic approach is that it requires a high number of individual measurements and that it can not be used as a direct witness.

The tomographic reconstruction is a process combin- ing information from many incompatible measurements in order to obtain quantities which are not measurable directly, such as off-diagonal elements of the density matrix. The elements of the reconstructed matrix contain, in principle, full information about the state. However, the information is practically limited due to unavailability of a complete set of measurement and the need to form prior assumptions about the suitable Hilbert space of the system [10]. For this reason some elements, in particular those related to higher Fock numbers, are difficult to estimate with sufficient precision. This may lead to erroneous or indeterminate conclusions, such as attributing nonclassical properties to interference of classical signals. Furthermore, the full information provided by the costly tomographic process is often not needed. In this case, use of a specialized and less demanding direct witness with straightforward statistical error analysis is much more effective. [11]

Two-photon interference leads to a state which, by suitably conditioning homodyne measurement, can be transformed into a state with quadrature squeezing [7]. This squeezing, although not strong enough to be of much use on its own, can be created neither from classical light nor from non-interfering photons and is therefore an excellent witness of nonclassical and coherence properties. It can be also directly measured. Interestingly, the measurement itself relies only on classical correlations within the state, without exploiting its entanglement [12] or discord [13]. The general witness therefore requires one further condition - the phase of the initial states needs to be randomized. This step, while not affecting single photon states, cancels the possible false positives. The full phase sensitive Hong-Ou-Mandel effect therefore serves as a directly observable witness simultaneously for both particle and wave-like aspects of the two photon interference. It also offers a new, much more challenging, benchmark against which new single photon sources can be tested. 
In this paper we introduce a witness for remotely prepared single photon states, which summarily assesses their single photon nature, indistinguishability, and compatibility with hybrid processing circuits. The witness relies on observation of nonclassical quadrature correlations and thus requires only measuring a single twoquadrature joint probability distribution, which makes it significantly less demanding than quantum tomography. For demonstration we apply the witness to a pair of photons individually prepared in timing synchronization cavities [8].

\section{SINGLE PHOTON WITNESS}

In the ideal version of the Hong-Ou-Mandel experiment, the two single photons indistinguishable in all but a single degree of freedom are in the initial state $|1,1\rangle$ and get mixed on a balanced beam splitter. This produces an entangled state

$$
|\psi\rangle=\frac{1}{\sqrt{2}}\left(|20\rangle-e^{i \phi}|02\rangle\right),
$$

with $\phi$ being the relative phase between the two output modes introduced by the beam splitter. The two resulting modes are then subjected to intensity measurements by avalanche photo diodes (APDs) whose action is described by their POVM elements $\Pi_{0}=|0\rangle\langle 0|$ and $\Pi_{1}=1-\Pi_{0}$, which provide us with probabilities $P_{k j}=\left\langle\psi\left|\Pi_{k} \otimes \Pi_{j}\right| \psi\right\rangle, k, j=0,1$. For indistinguishable photons there is a distinct lack of coincidences represented by $P_{11}=0$. On the other hand, for completely distinguishable photons the probability of coincident detection is firmly set to $P_{11}=1 / 2$. The full Hong-OuMandel measurement is realized by deliberately delaying one of the photons, thus increasing the distinguishability, and measuring the coincidence rates for these different settings. The effect is then quantified in terms of visibility, which is defined as

$$
V=\frac{\max P_{11}-\min P_{11}}{\max P_{11}+\min P_{11}}
$$

where the maximization and minimization is performed over the set of different delays, and it is equal to one for perfectly indistinguishable single photons and zero for perfectly distinguishable ones. The visibility can be also reduced due to imperfection of the single photon states, caused by presence of vacuum or higher Fock terms. However, since the vacuum terms do not contribute towards coincidence rates, only the higher Fock terms lead to reduction in visibility. This means the Hong-OuMandel effect is a good indicator of distinguishability of single photons, but it provides a limited insight into their quality. Another shortcoming of the Hong-Ou-Mandel effect is related to the technological aspect of the employed single photon detectors. These particle detectors have a very broad detection spectrum incompatible with the much narrower spectrum of continuous homodyne detectors, which play crucial role in CV quantum information processing with light [3]. This discrepancy can be removed by using optical filtering, but this comes with the cost of detection efficiency and alteration of the photon statistics. Another issue is the fundamental identity of the single photon detectors as intensity detectors. As such, the measurement is insensitive to the vacuum and can not reveal the wave portion of the interference - the relative phase between the modes - related to the phase sensitive correlations between the field quadratures. As a consequence, with the particle detectors the state (1) can not be differentiated from a completely mixed state $(|20\rangle\langle 20|+| 02\rangle\langle 02|) / 2$. Needless to say, in CV and hybrid quantum information processing this distinction is quite crucial.

We are therefore looking for a strong unifying witness capable of verifying the quality of single photon states as well as their indistinguishability and suitability for hybrid processing $[4,5]$. We also want to observe the interference effect directly, without the need to consider different measurement bases and settings, and subsequent reconstruction of the state's density matrix.

All of these requirements are reconciled in a three step procedure illustrated in Fig. 1a, in which the two single photon states are first phase randomized, then they interfere on a balanced beam splitter, and finally they are subjected to the joint measurement of phase-correlated quadrature operators $X_{1}\left(\theta_{1}\right)$ and $X_{2}\left(\theta_{2}\right)$, where $X_{j}(\theta)=$ $X_{j} \cos \theta+P_{j} \sin \theta$ with $j=1,2 . X_{j}$ and $P_{j}$ obey canonical commutation relation $\left[X_{j}, P_{j}\right]=i$. Because of the phase insensitivity of initial single photon states, a common component of phases has no significance. Hence, quadrature operators can be expressed only with the differential component $\Delta \theta=\theta_{1}-\theta_{2}$ as $X_{1}(\Delta \theta)$ and $X_{2}(0)$. Let us look at the ideal scenario with the state (1) and consider a homodyne measurement of mode 2 yielding a value $x_{2}$. This measurement projects the state of the remaining mode 1 into a state proportional to $e^{i \phi}\left\langle x_{2} \mid 2\right\rangle|0\rangle-\left\langle x_{2} \mid 0\right\rangle|2\rangle$. This state has zero mean values of the quadrature operators $X_{1}$ and $P_{1}$. The remarkable thing is that by choosing some particular values of $x_{2}$, the state in mode 1 is projected into a squeezed state, whose second moment at a certain measurement angle shows a value less than $1 / 2[7,14]$. The witness, confirming that state $\rho$ was obtained by interference of two phase insensitive single photon states on a balanced beam splitter, can be therefore formulated as

$$
E\left[X_{1}^{2}(\Delta \theta) \mid X_{2}(0)=x_{2}\right]=\frac{\operatorname{Tr}\left[X_{1}^{2}(\Delta \theta) \otimes\left|x_{2}\right\rangle\left\langle x_{2}\right| \rho\right]}{\operatorname{Tr}\left[1 \otimes\left|x_{2}\right\rangle\left\langle x_{2}\right| \rho\right]}<\frac{1}{2},
$$

where $E[\cdot \mid C]$ represents conditional mean value of an operator when condition $C$ is satisfied. The phase $\Delta \theta_{\mathrm{sq}}$ that gives minimum second moment is tied with the phase of superposition $\phi$ as $\Delta \theta_{\mathrm{sq}}=-\phi / 2$. It is important that $\phi$ is arbitrarily controllable by classical phase locking, so $\Delta \theta_{\text {sq }}$ is known a priori and can be set to an arbitrary 
(a)

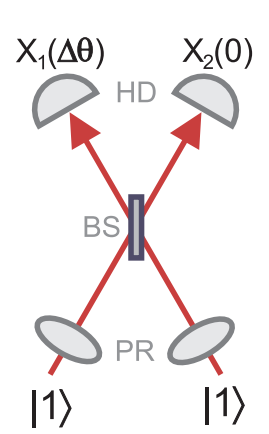

(b)

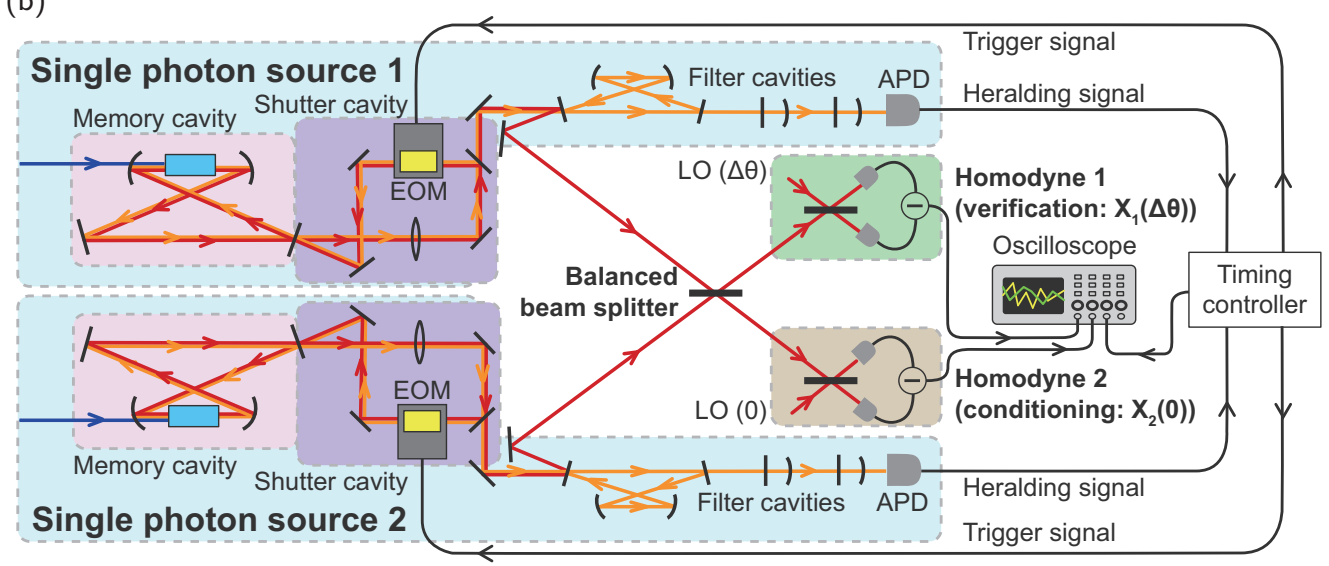

FIG. 1: (Color online) (a) - Conceptual scheme of the witness. The phase randomized (PR) states are mixed on a balanced beam splitter (BS) and two fixed quadratures are then measured by a pair of homodyne detectors (HD). (b) - Experimental setup. Two single photon states are independently generated and stored in the memory cavities. After synchronization, they are released at the same time, let to interfere on the balanced beam splitter, and recorded by the pair of homodyne detectors. EOM: electro-optic modulator, LO: local oscillator, APD: avalanche photo diode.

value. The procedure therefore requires measuring statistics of only a single pair of quadrature operators, which makes it much more practical and economical than the full tomography. Witness (3) is never satisfied when the states are distinguishable, classical, or incompatible with the CV part of the experiment. However, presence of other Fock components can diminish the squeezing and change the range of conditioning values $x_{2}$ which reveal it. On the other hand, when the squeezing threshold is reduced below vacuum level, the witness becomes stricter until, eventually, can be satisfied only by pure perfectly interfering single photon states. This is in greater detail discussed in the Appendix.

The conditioning on $x_{2}$ is related to the generalized theory of squeezing extraction presented in Ref. [7], but there are some interesting distinctions. Due to the ideally vanishing nature of the first moments, the condition can be specified with help of the second noncentral moment which makes it unsatisfiable by a lone single photon [15]. This is in contrast to observing squeezing of the conditional central moment, the variance, which can be achieved even for a single photon interacting with the vacuum and may therefore lead to false positives. However, the conditional squeezing can be considered as a witness for quality of single photons [7]. For example, an imperfect single photon state represented by density operator $\rho=\eta|1\rangle\langle 1|+(1-\eta)| 0\rangle\langle 0|$ can be confirmed as nonclassical for an arbitrary positive value of $\eta$. The method demonstrates phase-sensitive continuous variable nature of the correlations in photon anticorrelation experiments. It is also interesting to point out that when the squeezing is replaced by another nonclassicality witness employing only single quadrature measurement, such as one proposed in Ref. [16], no conditional nonclassicality can be detected.

\section{THE EXPERIMENTAL TEST}

The experimental setup for the test of the phasesensitive Hong-Ou-Mandel effect for two single photon states is based on our former experiment reported in Ref. [8] and it is shown in Fig. 1b. Each of the two photon sources is based on the heralded scheme with parametric down conversion contained in an optical cavity. Photon pairs are generated from the continuous-wave pump light at $430 \mathrm{~nm}$ at random times. One photon of the pair immediately leaves the cavity and is detected by an avalanche photodiode, thus heralding presence of another $860 \mathrm{~nm}$ photon stored in the cavity. A technique involving concatenated shutter cavity $[17,18]$ can be then used to release the second photon at a desired time. This allows timing synchronization of the two generated photons and enables their interference on the balanced beam splitter. With this technique, the simultaneous emission events occurs 20 times in a second with 400 ns synchronization window and we gathered quadrature data of 13,037 events. Since the single photon states are generated independently, their phase is random and needs no further randomization. The state after interference is measured by phase-sensitive homodyne detection in both output modes of the balanced beam splitter: one mode is used for conditioning to generate squeezing and the other mode is used for the verification of squeezing in the conditioned state. The relative phase between a local oscillator and the output mode at both homodyne detections, and the relative phase of two local oscillators which is equivalent to $\Delta \theta$ are actively stabilized. The quantum efficiency of homodyne detectors and the visibility between a signal beam and the local oscillator are both more than $99 \%$. Any correction of losses is not applied to the results below.

Fig. 2 (a) shows the joint measured distribution of 
(a)

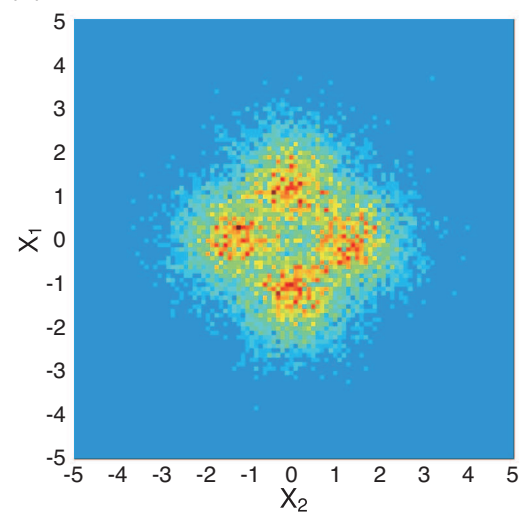

(b)

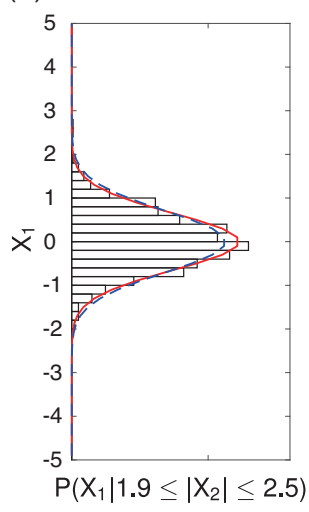

FIG. 2: (Color online) Experimentally measured quadrature distribution of the state after interference with $\Delta \theta=\Delta \theta_{\text {sq }}$. (a) Joint probability distribution $P\left(X_{1}, X_{2}\right)$. (b) Normalized quadrature distribution $P\left(X_{1}\right)$. An occurrence rate of $X_{1}$ with the window $1.9 \leq\left|X_{2}\right| \leq 2.5$ is shown in histogram. Lines are Gaussian distributions with the variance of conditioned $P\left(X_{1}\right)$ (solid red) and that of vacuum fluctuation for reference (dashed blue), respectively.

quadratures $X_{1}$ and $X_{2}$ of the two modes emerging from the beam splitter with $\Delta \theta=\Delta \theta_{\mathrm{sq}}$. The quadratures were locked against each other, but their relation to the outside reference varied over time. This effectively resulted in a statistics which is equivalent to measurement without an outside phase reference. We will therefore omit the phase dependence from now on. We can see that the quadrature data are distinctively correlated in a manner consistent with the target state. In accordance with the proposed witness, we are looking for a quantitative indicator of the Hong-Ou-Mandel coherence in the form of the second conditional moment $E\left[X_{1}^{2} \mid X_{2}=x_{2}\right]$. The theory asks for conditioning on a single measurement result. Since this is impossible, we have relaxed the requirement for condition and accepted situations in which the value of quadrature $X_{2}$ fell into a bin of width $\Delta, x_{2}-\Delta / 2 \leq X_{2} \leq x_{2}+\Delta / 2$. Due to the symmetrical nature of the distribution, we also used both positive and negative values in the post-selection. An example of the conditional quadrature distribution of $X_{1}$ is shown in Fig. 2 (b). The conditioning window, denoted by set $B$, was set to $1.9 \leq\left|X_{2}\right| \leq 2.5$ and it picked 1,011 of the initial 13,037 data points. The conditional variance was found to be $E\left[X_{1}^{2} \mid X_{2} \in B\right]=0.42$, which is smaller than the vacuum fluctuations variance of 0.5 .

Let us now discuss the results in more detail, giving more attention to possible statistical errors, and make sure the observed squeezing is significant. For that we need to construct the confidence band for the estimated variance. Under the assumption that the conditional distributions are approximately Gaussian we used Monte Carlo method to simulate 1,000 runs of the experiment, estimated their moments and evaluated their statistics. The standard deviation of the estimated variances de- pends on the number of data points and therefore on the position and the width of the post-selection interval. A narrow interval generally yields the lowest possible moment values, but they come at the cost of low number of data points and, consequently, lesser certainty about the validity of the result. The best certifiable violation is obtained when the upper boundary of the confidence band reaches its minimum value. Fig. 3 shows the second moment with its upper confidence band estimated from the measured data. For all considered values of $\Delta$ we have optimized over the values $x_{2}$ marking the center of the interval to obtain the minimum of the upper confidence band boundary. The plotted moment can be therefore expressed as

$E_{\min , \Delta}\left[X_{1}^{2}\right]=\min _{x_{2}}\left\{E\left[X_{1}^{2}\left|x_{2}-\frac{\Delta}{2}<\right| X_{2} \mid<x_{2}+\frac{\Delta}{2}\right]\right\}$.

We have obtained the lowest certifiable variance for $\Delta=$ 0.6 .

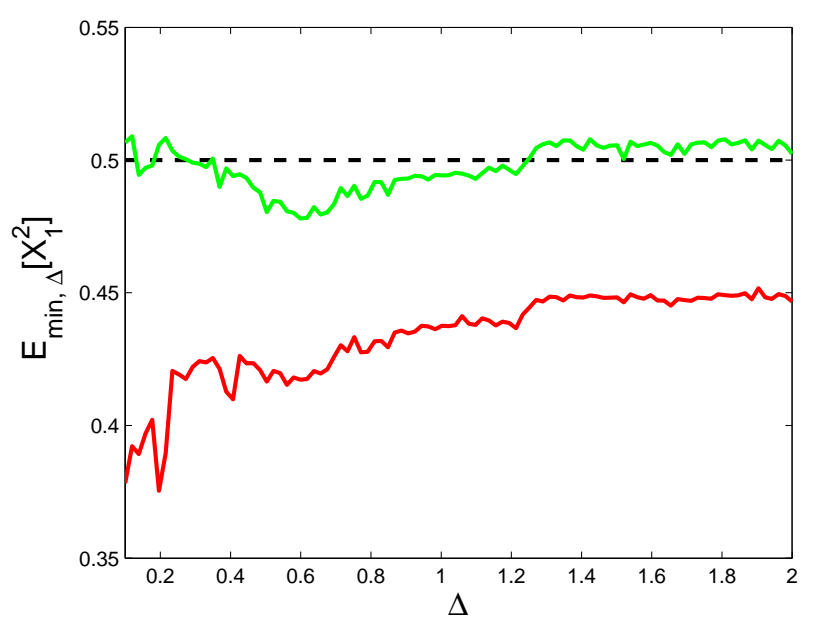

FIG. 3: (Color online) The conditioned second moment $E_{\min , \Delta}\left[X_{1}^{2}\right]$ (red) and its upper confidence band (green) relative to the width of the postselection interval $\Delta$. The position of the conditioning interval given by the measured value of $X_{2}$ was optimized over in order to obtain minimal certifiable variance given by the upper confidence interval.

The conditional variances for this scenario are shown in Fig. 4. We can see that the measured data closely follow the curve predicted from knowledge of the two independent single photon states, which was obtained by their individual tomographic reconstructions [8]. This shows that the amount of squeezing is limited mainly by the undesired vacuum component of the single photons and not their coherence. Furthermore, the squeezing is observable even if we take the three standard deviations wide confidence band into account. This confirms that the squeezing is not a statical artifact and demonstrates both the nonclassical nature of the two initial single photon states and their mutual coherence. This first application of the proposed witness also shows its sensitivity 
to small imperfection of these components. Note, visibility of the traditional Hong-Ou-Mandel interference estimated from the tomography data would be $V=0.7$ in our experiment.

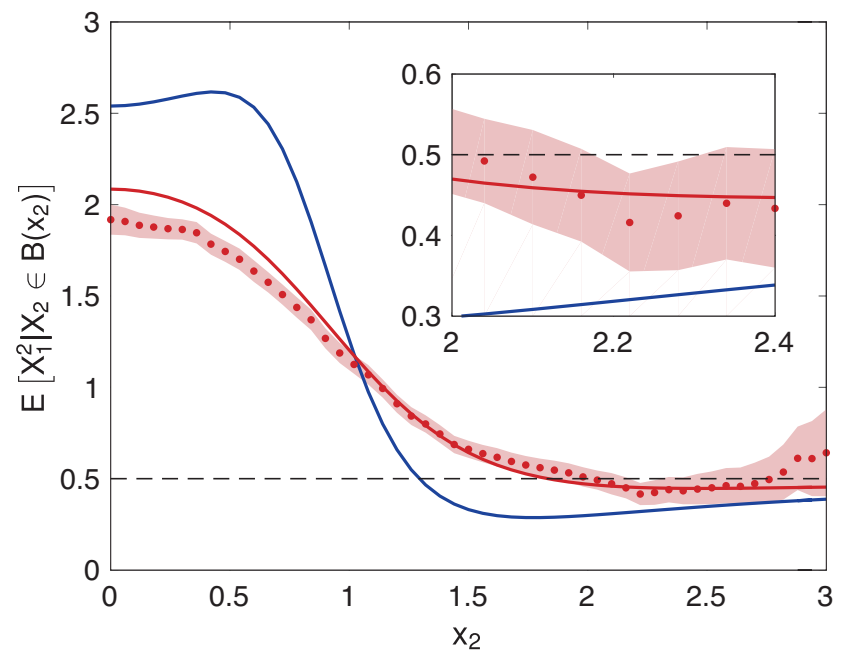

FIG. 4: (Color online) The conditional second moment $E\left[X_{1}^{2} \mid X_{2} \in B\left(x_{2}\right)\right]$ where $B\left(x_{2}\right)$ is the set of values satisfying $x_{2}-\Delta / 2<\left|X_{2}\right|<x_{2}+\Delta / 2$. The width of the post-selection interval was chosen to be $\Delta=0.6$. Dashed blue line marks theoretical prediction for the interference of two ideal single photon states. Solid red line shows prediction for interference of two imperfect photons produced by the cavities. Red dots represent experimental data with the light red zone marking the $3 \sigma$ confidence band. The inset shows detailed behavior in the area with the highest certifiable squeezing.

\section{SUMMARY}

In summary, we have suggested a phase sensitive HongOu-Mandel effect as a witness for recognizing presence and non-classicality of individual photons, their ability to quantum mechanically interfere, and their compatibility with hybrid quantum processing [3]. These aspects are detected jointly, lack of any one of them will lead to diminishing performance and separating them is not straightforward. However, since all of these properties are needed for hybrid processing, the ability to test them at the same time is highly practical. The suggested witness requires joint measurement of only a single pair of commuting quadrature operators. There is no need for tomography and sets of incompatible measurements, which reduces the demands on length of the measurement by more than an order of magnitude. The employed measurement does not rely on entanglement [12] or discord [13]. We have applied the witness to the pair of on demand generated photons and demonstrated both their nonclassical nature and their mutual coherence. The witness can be directly applied to other experimental platforms where generation of interfering Fock states plays an important role, like atomic ensembles [19], optomechanics [20, 21], and superconducting circuits [22, 23].

\section{Acknowledgement}

This work was partly supported by CREST of JST, JSPS KAKENHI, APSA. P. M., P.Z., and R. F. acknowledge Project GB14-36681G of the Czech Science Foundation. Y. H. acknowledges support from ALPS. K. M. acknowledges support from JSPS.

\section{Appendix}

The proposed witness takes advantage of nonclassical properties of the interfering quantum states. As such, higher Fock components of the initial states, which are nonclassical on their own, can affect the outcome. Since we consider phase randomized states with density matrix diagonal in Fock representation, $\rho_{1,2}=\sum_{k} P_{k}|k\rangle\langle k|$, we can analyze the effect by looking at the witness value for initial states $|m, n\rangle$, because the final witness value will be obtained as their linear combination. The conditional second moments for states $|m, n\rangle$, where $m, n=0,1,2$, are displayed in Fig. 5. We can see that states $|1,0\rangle$ and $|2,0\rangle$ never lead to squeezing and numerics confirmed this behavior for states up to $|10,0\rangle$. This demonstrates that even when the two states are imperfect their coherence is an absolutely essential condition for observation of any squeezing.

We can also see that apart from state $|1,1\rangle$ squeezing can be also obtained for states $|1,2\rangle$ and $|2,2\rangle$. However, the minimum conditioning value, for which the squeezing is observable, is larger than that of the state $|1,1\rangle$. Combination of higher Fock terms have not been plotted, but the trend remains the same: for some states squeezing can be observed but it is always for progressively larger values of $x_{2}$. This can be used for deriving even a stronger form of the witness, which eliminates contributions of these higher terms. For example, squeezing found for conditioning value $1.23<x_{2}<1.75$ can be only consequence of the state $|1,1\rangle$.

For explicit demonstration, let us consider a subset of states with at most two photons. Such states have density matrix $\rho_{1}=\rho_{2}=p_{0}|0\rangle\left\langle 0\left|+p_{1}\right| 1\right\rangle\langle 1|+\left(1-p_{0}-\right.$ $\left.p_{1}\right)|2\rangle\langle 2|$. Fig. 6 shows range of parameters, in which squeezing can be detected, for two different conditioning values. $x_{2}=1.5$ corresponds to the strong ideal witness which detects only single photon contributions, while $x_{2}=2.2$ relates to the value which was used in the experimental implementation. We can see, that the first case restrictively singles out only states with high single photon component, while the second one allows also for two-photon contribution. However, the second one is also more forgiving when it comes to losses causing higher proportion of the vacuum, which is the feature 
we can take advantage of if we admit the realistic experimental assumption that $p_{2} \ll 1$.

The amount of observed squeezing can be used for quantitative statements about quality of the single photons and their distinguishability. Lowering the required moment value reduces the range of parameters for which the behavior can be witnessed. Sufficiently low value then detects only pure perfectly interfering single photon states. Alternatively, larger observed squeezing allows for wider confidence intervals and thus for more valid statements.

In summary, observation of squeezing always guarantees that the two states are nonclassical and coherent. Observation of squeezing for particular values of $x_{2}$ can provide further information and even certify presence of specific Fock components.

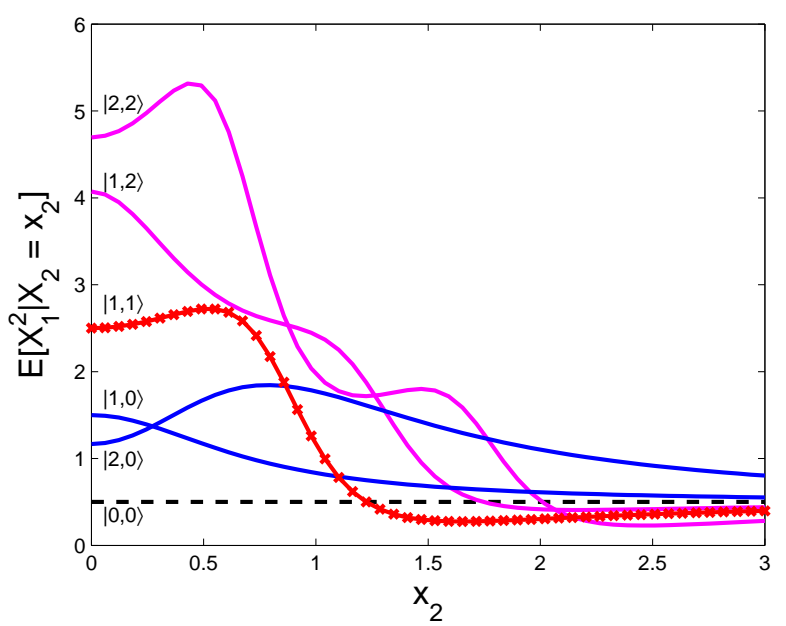

FIG. 5: (Color online) Conditional second moment for different input states.

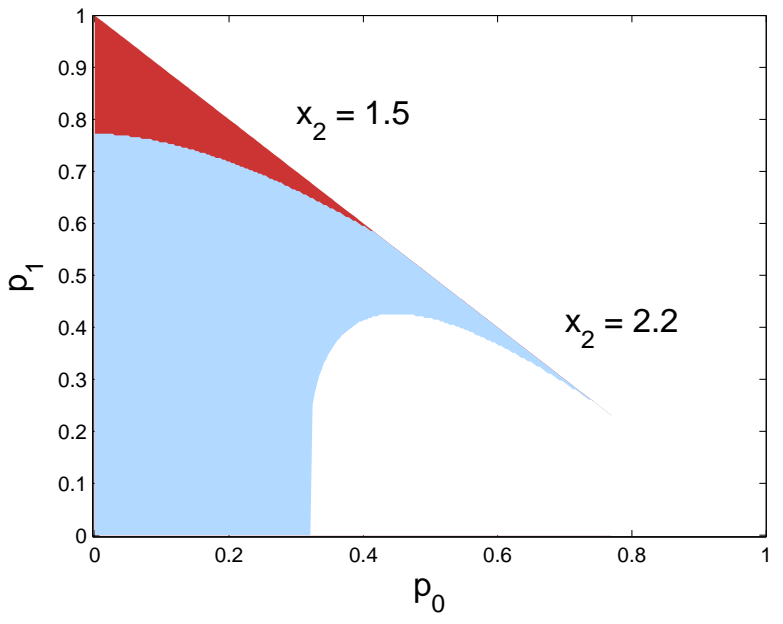

FIG. 6: (Color online) Range of parameters for states $\rho_{1}=$ $\rho_{2}=p_{0}|0\rangle\left\langle 0\left|+p_{1}\right| 1\right\rangle\left\langle 1\left|+p_{2}\right| 2\right\rangle\langle 2|$, in which squeezing can be witnessed. For conditioning value $x_{2}=2.2$ squeezing can be observed in both the light blue and the dark red areas. For conditioning value $x_{2}=1.5$ squeezing can be observed only in the dark red area. 
[1] C. K. Hong, Z. Y. Ou, and L. Mandel, Measurement of subpicosecond time intervals between two photons by interference, Phys. Rev. Lett. 59, 2044 (1987).

[2] T. Kobayashi, R. Ikuta, S. Yasui, S. Miki, T. Yamashita, H. Terai, T. Yamamoto, M. Koashi, and N. Imoto, Frequency-domain Hong-Ou-Mandel interference, Nature Photonics 10, 441 (2016); R. Lopes, A. Imanaliev, A. Aspect, M. Cheneau, D. Boiron, and C. I. Westbrook, Atomic Hong-Ou-Mandel experiment, Nature 520, 66 (2015); K. Toyoda, R. Hiji, A. Noguchi, and S. Urabe, Hong-Ou-Mandel interference of two phonons in trapped ions, Nature 527, 74 (2015); A. M. Kaufman, B. J. Lester, C. M. Reynolds, M. L. Wall, M. Foss-Feig, K. R. A. Hazzard, A. M. Rey, and C. A. Regal, Two-particle quantum interference in tunnel-coupled optical tweezers Science 345, 306 (2014); A. Sipahigil, K. D. Jahnke, L. J. Rogers, T. Teraji, J. Isoya, A. S. Zibrov, F. Jelezko, and M. D. Lukin, Indistinguishable photons from separated silicon-vacancy centers in diamond, Phys. Rev. Lett. 113, 113602 (2014).

[3] U. L. Andersen, J. S. Neergaard-Nielsen, P. van Loock, and A. Furusawa, Hybrid discrete- and continuousvariable quantum information, Nature Physics 11, 713 (2015).

[4] A. Ourjoumtsev, H. Jeong, R. Tualle-Brouri, and P. Grangier, Generation of optical 'Schrödinger cats' from photon number states, Nature 448, 784 (2007).

[5] J. Etesse, M. Bouillard, B. Kanseri, and R. Tualle-Brouri, Experimental generation of squeezed cat states with an operation allowing iterative growth, Phys. Rev. Lett. 114, 193602 (2015).

[6] A. E. Ulanov, I. A. Fedorov, D. Sychev, P. Grangier, and A. I. Lvovsky, Loss-tolerant state engineering for quantum-enhanced metrology via the reverse HongOu-Mandel effect, Nature Communications 7, 11925 (2016); H. Jeong, A. Zavatta, M. Kang, S.-W. Lee, L. S. Costanzo, S. Grandi, T. C. Ralph, and M. Bellini, Generation of hybrid entanglement of light, Nature Photonics 8, 564 (2014).

[7] R. Filip and P. Zapletal, Squeezed-state generation from single-photon sources, Phys. Rev. A 90, 043854 (2014).

[8] K. Makino, Y. Hashimoto, J. Yoshikawa, H. Ohdan, T. Toyama, P. van Loock, and A. Furusawa, Synchronization of optical photons for quantum information processing, Science Advances 2, e1501772 (2016).

[9] M. Cooper, E. Slade, M. Karpiński, and B. J. Smith, Characterization of conditional state-engineering quantum processes by coherent state quantum process tomography, New J. Phys. 17, 033041 (2015).

[10] Y. S. Teo, H. Zhu, B.-G. Englert, J. Řeháček, and Z. Hradil, Quantum-State Reconstruction by Maximizing Likelihood and Entropy, Phys. Rev. Lett. 107, 020404 (2011).
[11] A. K. Ekert, C. M. Alves, D. K. L. Oi, M. Horodecki, P. Horodecki, and L. C. Kwek, Direct Estimations of Linear and Nonlinear Functionals of a Quantum State, Phys. Rev. Lett. 88, 217901 (2002); R. Filip, Overlap and entanglement-witness measurements, Phys. Rev. A 65, 062320 (2002); K. L. Pregnell, Measuring Nonlinear Functionals of Quantum Harmonic Oscillator States, Phys. Rev. Lett. 96, 060501 (2006).

[12] R. Horodecki, P. Horodecki, M. Horodecki, and K. Horodecki, Quantum entanglement, Rev. Mod. Phys. 81, 865 (2009).

[13] K. Modi, A. Brodutch, H. Cable, T. Paterek, and V. Vedral, The classical-quantum boundary for correlations: Discord and related measures, Rev. Mod. Phys. 84, 1655 (2012).

[14] For example, for $\theta=0$ conditioning $x_{2}=\sqrt{2}$ produces state $(3|0\rangle-\sqrt{2}|2\rangle) / \sqrt{11}$ with $\left\langle X^{2}\right\rangle=7 / 22<0.5$.

[15] Single photon divided on a beam splitter and conditioned by homodyne detection can be always expressed as $c_{0}|0\rangle+$ $c_{1}|1\rangle$. In this state, $\left\langle X^{2}\right\rangle=0.5+\left|c_{1}\right|^{2}$ and the moment is never squeezed.

[16] W. Vogel, Nonclassical states: an observable criterion, Phys. Rev. Lett. 84, 0031 (2000).

[17] J. Yoshikawa, K. Makino, S. Kurata, P. van Loock, and A. Furusawa, Creation, storage, and on-demand release of optical quantum states with a negative Wigner function, Phys. Rev. X 3, 041028 (2013).

[18] J. Yoshikawa, K. Makino, A. Furusawa, in Engineering the Atom-Photon Interaction, A. Predojević, M. W. Mitchell, Eds. (Springer Cham, 2015), chap. 8.

[19] M. Ebert, A. Gill, M. Gibbons, X. Zhang, M. Saffman, and T. G. Walker, Atomic Fock state preparation using Rydberg blockade, Phys. Rev. Lett. 112, 043602 (2014).

[20] A. D. O'Connell, M. Hofheinz, M. Ansmann, R. C. Bialczak, M. Lenander, E. Lucero, M. Neeley, D. Sank, H. Wang, M. Weides, J. Wenner, J. M. Martinis, and A. N. Cleland, Quantum ground state and single-phonon control of a mechanical resonator, Nature 464, 697 (2010).

[21] C. Galland, N. Sangouard, N. Piro, N. Gisin, and T. J. Kippenberg, Heralded single-phonon preparation, storage, and readout in cavity optomechanics, Phys. Rev. Lett. 112, 143602 (2014).

[22] M. Hofheinz, E. M. Weig, M. Ansmann, R. C. Bialczak, E. Lucero, M. Neeley, A. D. O'Connell, H. Wang, J. M. Martinis, and A. N. Cleland, Generation of Fock states in a superconducting quantum circuit, Nature 454, 310 (2008).

[23] S. P. Premaratne, F.C. Wellstood, and B.S. Palmer, Microwave photon Fock state generation by stimulated Raman adiabatic passage, Nature Communications 8, 14148 (2017). 